\title{
Prevalent cerebrovascular and cardiovascular disease in people with Parkinson's disease: a meta-analysis
}

This article was published in the following Dove Press journal:

Clinical Epidemiology

\author{
Chien Tai Hong ${ }^{1,2}$ \\ Han-Hwa $\mathrm{Hu}^{1,2}$ \\ Lung Chan ${ }^{1,2, *}$ \\ Chyi-Huey Bai, ${ }^{3,4, *}$
}

'Department of Neurology, Shuang-Ho Hospital, Taipei Medical University, New Taipei City, Taiwan: 2Department of Neurology, School of Medicine, College of Medicine, Taipei Medical University, Taipei City, Taiwan; ${ }^{3}$ School of Public Health, College of Public Health, Taipei Medical University, Taipei City, Taiwan; ${ }^{4}$ Department of Public Health, College of Medicine, Taipei Medical University, Taipei City, Taiwan

*These authors contributed equally to this work
Correspondence: Chyi-Huey Bai School of Public Health, College of Public Health, Taipei Medical University, 5/F Health Science Building, $250 \mathrm{Wu}-\mathrm{Hsing}$ Street, Taipei City I I0, Taiwan Tel +886 22736 I66I ext65I0

Fax +88622738 483।

Email baich@tmu.edu.tw

Lung Chan

Department of Neurology, Shuang-Ho Hospital, Taipei Medical University, No. 29I, Zhongzheng Road, Zhonghe District, New Taipei City 23561, Taiwan

Tel +886222490088 ext8I I 2

Email cjustinmd@gmail.com
Background: People with Parkinson's disease (PwP) are speculated to be at a low risk of cerebrovascular and cardiovascular disease (CVD) because they have fewer vascular risk factors and lower smoking rate. However, emerging evidence suggests that $\mathrm{PwP}$ are at higher risk of CVD, which introduces controversy to the notion that there is no association between Parkinson's disease (PD) and CVD. Hence, we conducted a meta-analysis to analyze the risk of CVD in PwP. Methods: Electronic databases were searched using terms related to PD and CVD. Articles were included in the meta-analysis only if they employed clear diagnostic criteria for PD and CVD. The reference lists of the relevant articles were reviewed to identify eligible studies not found during the keyword search.

Results: The enrolled studies were categorized into case-control and cohort studies, and the former was further divided into postmortem (three) and clinical (four) studies. In the clinical case-control studies group, PD was more associated with CVD (OR: 2.89, 95\% CI: 1.36-6.13). Three studies were enrolled in the cohort studies group, and the merged results demonstrated that PwP were at higher risk of CVD during the follow-up period (HR: 1.84, 95\% CI: 1.34-2.54). Conclusion: $\mathrm{PD}$ is associated with CVD, which may be due to the shared pathogeneses between the two diseases or PD-related effects. PwP should be more aware of the risk of CVD despite having fewer traditional vascular risk factors.

Keywords: Parkinson's disease, cerebrovascular disease, cardiovascular disease, meta-analysis, case-control, cohort, cerebral multimorbidity

\section{Introduction}

Parkinson's disease (PD) is the second most common neurodegenerative disease. ${ }^{1}$ The pathological hallmark of PD is the degeneration of dopaminergic neurons in the midbrain substantia nigra, which results from the combination of multiple pathogeneses, including mitochondrial dysfunction, $\alpha$-synuclein aggregation, and neuroinflammation. ${ }^{2}$ According to the UK Parkinson's Disease Society Brain Bank clinical diagnostic criteria, rigidity, bradykinesia, tremor, and postural instability are the key features of PD. ${ }^{3}$ Patients with a history of repeated strokes and stepwise progression of parkinsonism symptoms are more likely to be categorized as having vascular parkinsonism (VP) and should be excluded from diagnosis of PD. Clinically, VP is distinguished from PD by the presence of lower body-predominate parkinsonism and the poor response to dopaminergic medications. ${ }^{4}$

People with Parkinson's disease (PwP) are speculated to be at a low risk of cerebrovascular and cardiovascular disease (CVD) due to their lower smoking rate, ${ }^{5,6}$ fewer 
vascular risk factors, ${ }^{7}$ and the exclusion of VP at diagnosis. However, postmortem studies have demonstrated that PwP and controls exhibit identical rates of overall cerebrovascular diseases, except fewer symptomatic ones. ${ }^{8-11}$ Furthermore, recent large-scale cohort studies have demonstrated that $\mathrm{PD}$ is associated with higher risk of CVD. ${ }^{12-14}$ This discrepancy challenges the previous notion that PD is inversely associated with CVD.

Due to the controversy regarding the association between PD and CVD and the varying results from different studies, a meta-analysis is warranted to clarify this relationship. Based on the differences in study designs, we separated the enrolled articles into case-control and cohort studies, and the former was further divided into postmortem and clinical studies to investigate the association between the two diseases.

\section{Methods}

\section{Literature search strategy}

All relevant articles published in English between January 1, 1990, and May 31, 2017, were identified by searching PubMed, BioMed Central, MEDLINE, and Google Scholar. The keywords used for the search were cohort study[MeSH], case control study[MeSH], Parkinson, Parkinson's, Parkinson's disease, cardiovascular diseases/ event, coronary disease/event, cerebrovascular diseases/ event, stroke, myocardial infarction, ischaemial, and their combinations. The detailed keywords are presented in Table $\mathrm{S} 1$. The reference lists of the relevant articles were reviewed to identify eligible studies that were not found using these search keywords.

\section{Inclusion and exclusion of literature}

The inclusion criteria were as follows: 1) clear definition of PD diagnosis - there must be a description about the clinical or pathological PD diagnostic criteria used in the study, and the criteria must be well acknowledged (i.e. pathology, UK Parkinson's Disease Society Brain Bank criteria, or Gelb criteria) or validated (i.e. the diagnosis of PD in General Practice Research Database through Oxford Medical Information System or READ-code); ${ }^{15}$ 2) clear definition of CVD diagnosis - there must be a description about the methods to define CVD, including disease claim coding system, CVD record from the medical chart, consensual radiological findings, and pathological findings; 3 ) cohort study or case-control study published as an original article, case series, or letter to the editor; 4) studied population must have included $\geq 50$ persons; and 5) publication in English. We generated a long list of all the papers (156 case-control studies and 242 cohort studies). The articles were excluded when all of the three committee members (Bai C-H, Hong $\mathrm{CT}$, and Chan L) rejected the abstracts. 25 case-control studies and 32 cohort studies were shortlisted, and their full text was reviewed independently. A study was included/excluded if all the committee members agreed/disagreed. Decisions of inclusion or exclusion of other studies were based on a committee meeting. Finally, only seven case-control studies (three postmortem and four clinical studies) and three cohort studies were included in the meta-analysis.

\section{Data extraction}

The following data were extracted: first author name, publication year, country and location where the study was conducted, study design, and the diagnostic criteria for stroke and PD. All data were independently reviewed by three investigators (Bai C-H, Hong CT, and Chan L), and any disagreements were resolved through consensus. Data from the 10 candidate articles were extracted by Bai C-H.

\section{Statistical analysis}

The significance of each pooled OR and pooled HR was determined using a $Z$-test, in which $p<0.05$ was considered to indicate a significant difference. The $\chi^{2}$-based $Q$ statistical test and $I^{2}$ test were used to assess the heterogeneity across studies. In the analyses, if the heterogeneity was low, we used a fixed-effects model; otherwise, we applied a randomeffects model. Review Manager 5.3 software (available from Cochrane) was used to perform the meta-analyses. All statistical analyses were reviewed using SAS software (version 9.3; SAS Institute Inc., Cary, NC, USA). All reported $p$ values were two-sided, with $p<0.05$ considered statistically significant.

\section{Results}

Figure 1 summarizes the process of identifying the eligible case-control (Figure 1A) and cohort studies (Figure 1B). In total, 156 case-control and 242 cohort full-text articles that were published between January 1, 1990, and May 31, 2017, were selected for eligibility assessment. We excluded articles with unclear definitions of PD and CVD and followup details as well as articles irrelevant to our study topic. Finally, seven case-control (three postmortem ${ }^{8,10,11}$ and four clinical studies ${ }^{12,16-18}$ ) and three cohort articles ${ }^{12-14}$ satisfied the aforementioned criteria and were subjected to qualitative synthesis.

The characteristics of the seven case-control studies are summarized in Table 1. In total, $1548 \mathrm{PwP}$ and 1666 
A

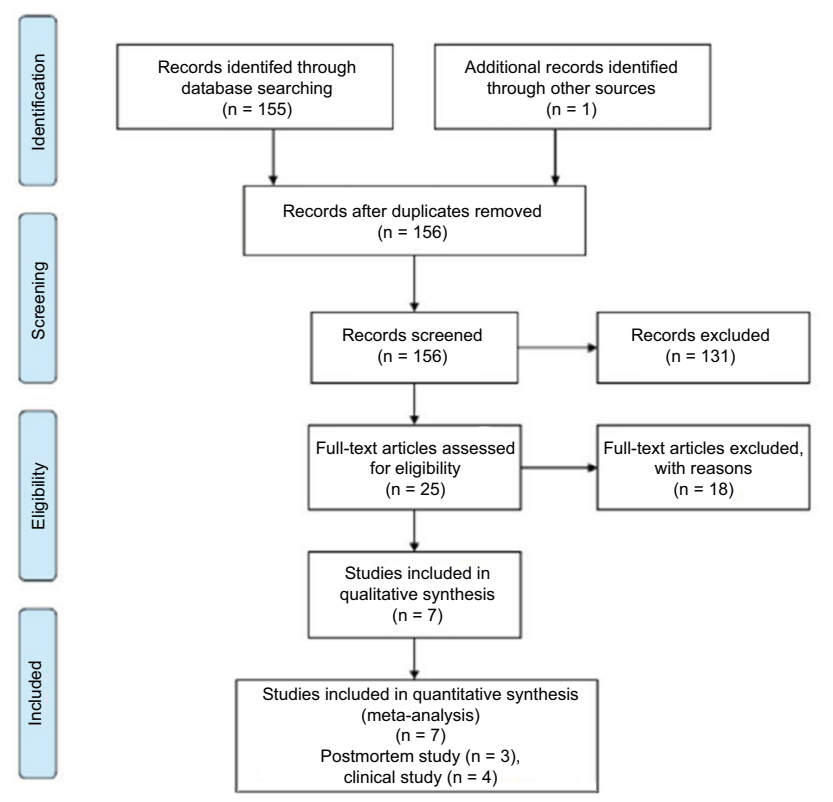

B

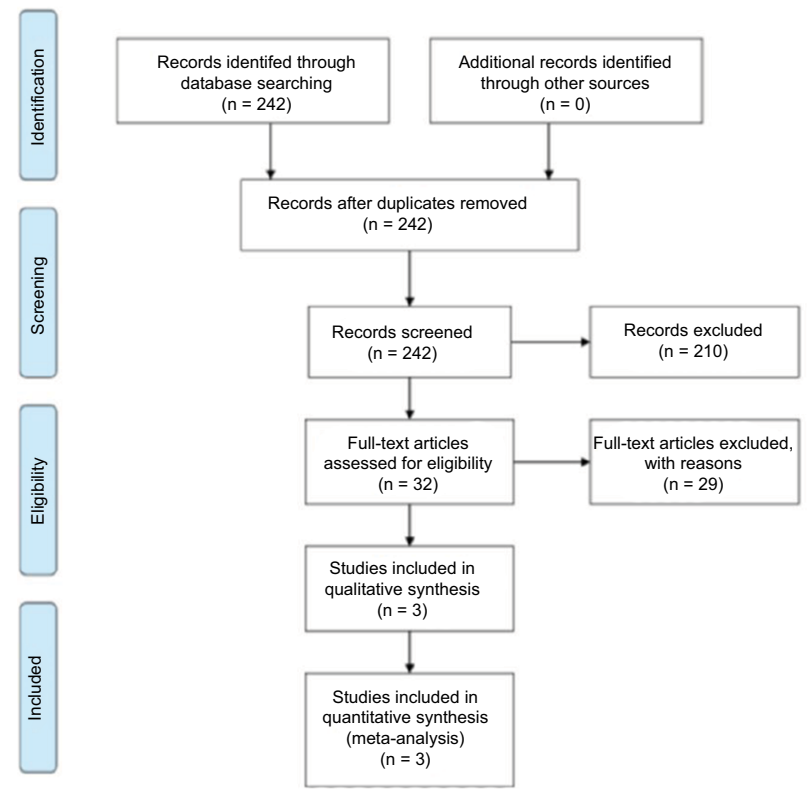

Figure I Flowchart of literature search. (A) Case-control studies. (B) Cohort studies.

Table I Summary of case-control studies

\begin{tabular}{|c|c|c|c|c|}
\hline Study & Country & PD diagnostic criteria & $\begin{array}{l}\text { Number of PD } \\
\text { cases/controls }\end{array}$ & CVD diagnostic tool \\
\hline Mastaglia et al" & Australia & Autopsy & $100 / 100$ & Pathology \\
\hline Jellinger ${ }^{10}$ & Austria & Autopsy & $200 / 200$ & Pathology \\
\hline Jellinger ${ }^{8}$ & Austria & Autopsy & $617 / 535$ & Pathology \\
\hline Becker et al ${ }^{12}$ & UK & $\begin{array}{l}\text { Oxford Medical Information System or } \\
\text { READ-code + exclusions }\end{array}$ & $128 / 494$ & $\begin{array}{l}\text { Medical information from health care } \\
\text { database }\end{array}$ \\
\hline Patel et al ${ }^{16}$ & UK & $\begin{array}{l}\text { UK Parkinson's Disease Society Brain Bank } \\
\text { Clinical Diagnosis Criteria for Parkinson's } \\
\text { Disease }\end{array}$ & $50 / 50$ & Neuroimaging \\
\hline Skeie et $\mathrm{al}^{17}$ & Norway & Gelb criteria & $212 / 175$ & Interviews on medical history \\
\hline Song et al ${ }^{18}$ & South Korea & $\begin{array}{l}\text { UK Parkinson's Disease Society Brain Bank } \\
\text { Clinical Diagnosis Criteria for Parkinson's } \\
\text { Disease }\end{array}$ & $241 / 112$ & Magnetic resonance imaging \\
\hline
\end{tabular}

Abbreviations: PD, Parkinson's disease; CVD, cardiovascular disease.

controls were enrolled in the studies. Five of the studies were conducted in Europe, one in Australia, and one in South Korea. Three out of the seven were autopsy studies, and the diagnosis of CVD and PD was based on pathology. In the rest of the clinical studies, the diagnosis of PD was based on UK Parkinson's Disease Society Brain Bank Clinical Diagnosis Criteria for Parkinson's Disease, Gelb criteria, the Oxford Medical Information System, or READ-code. Outcome measurements included self-reported history of stroke and transient ischemic attack (TIA), disease claim coding system, CVD record from the medical chart, radiological findings, or pathological findings.

Because of the huge difference in the nature of clinical and postmortem studies, the analysis of these two types of studies was done separately. Merging the data from the four clinical case-control studies, the OR of CVD history among PwP was 2.89 (95\% CI: 1.36-6.13) (Figure 2A). On the other hand, the OR of the presence of cerebral infarct pathology among PwP in the three postmortem studies was 1.15 (95\% CI: 0.92-1.44) (Figure 2B).

The characteristics of the included cohort studies are summarized in Table 2. Three cohort studies were enrolled, all of which used large-scale medical research databases: the Taiwan National Health Insurance Research Database and UK-based General Practice Research Database. The diagnosis of PD was based on the disease claim coding system. The outcomes were ischemic stroke in two studies, ischemic stroke and TIA in one study, and acute myocardial infarction 
A

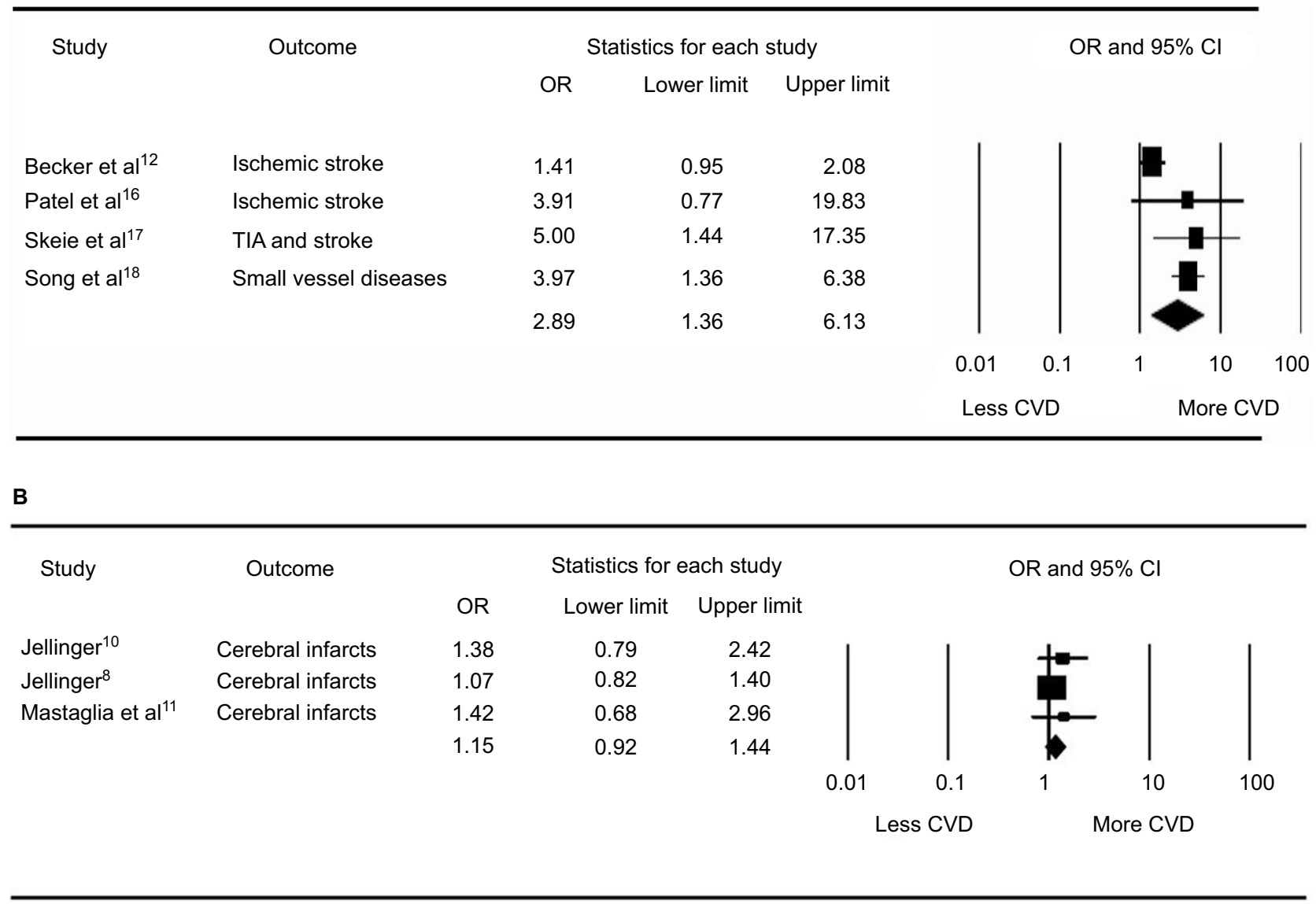

Figure 2 (A) Forest plot illustrating the risk of cerebrovascular disease/CVD among PwP from clinical case-control studies. (B) Forest plot illustrating the risk of cerebral infarcts among PwP from clinical postmortem case-control studies.

Abbreviations: CVD, cardiovascular disease; PwP, people with Parkinson's disease; TIA, transient ischemic attack.

Table 2 Summary of cohort studies

\begin{tabular}{|c|c|c|c|c|c|}
\hline Study & Country & PD diagnosis criteria & $\begin{array}{l}\text { Number of PD } \\
\text { cases/controls }\end{array}$ & CVD diagnostic tool & Median follow-up \\
\hline Becker et $\mathrm{al}^{12}$ & UK & $\begin{array}{l}\text { Oxford Medical Information System or } \\
\text { READ-code + exclusions }\end{array}$ & $2,553 / 2,127$ & $\begin{array}{l}\text { Medical information from } \\
\text { health care database }\end{array}$ & Did not provide \\
\hline Huang et $\mathrm{al}^{13}$ & Taiwan & $\begin{array}{l}\text { International Classification of Diseases claim } \\
+ \text { exclusion }\end{array}$ & $2,204 / 2,204$ & $\begin{array}{l}\text { Medical information from } \\
\text { health care database }\end{array}$ & 30.6 months \\
\hline Liang et $\mathrm{al}^{14}$ & Taiwan & $\begin{array}{l}\text { International Classification of Diseases claim } \\
+ \text { exclusion }\end{array}$ & $3,2|I / 3,2| I$ & $\begin{array}{l}\text { Medical information from } \\
\text { health care database }\end{array}$ & 29.0 months \\
\hline
\end{tabular}

Abbreviations: PD, Parkinson's disease; CVD, cardiovascular disease.

in one study. Regarding the temporal sequence of PD and CVD, because of the nature of the cohort study, CVD onset occurred after the diagnosis of PD. When the results of the three cohort studies were pooled together, the HR of CVD among PwP was 1.84 (95\% CI: 1.34-2.54) (Figure 3).

\section{Discussion}

This meta-analysis demonstrated that PD is associated with CVD, and PwP were at a higher risk of CVD later in their life. These results provide new evidence to address the controversy of the association between PD and CVD, which hints that PD may share some common pathogeneses with CVD, and PwP should be more alert about the risk of CVD.

Mitochondrial dysfunction and excessive reactive oxygen species (ROS) constitute the major pathogenesis of PD. ${ }^{2}$ Moreover, systemic mitochondrial dysfunction triggers atherosclerosis as well. Oxidative damage of mitochondrial DNA is correlated with the severity of atherosclerosis. ${ }^{19}$ Excessive ROS results in the destruction of pancreatic $\beta$-cells, increased oxidation of low-density lipoprotein, and dysfunction of endothelial cells, which promote atherosclerosis. ${ }^{20}$ These shared pathogeneses - mitochondrial dysfunction and 


\begin{tabular}{|c|c|c|c|c|c|c|c|}
\hline Study & Outcome & \multicolumn{3}{|c|}{ Statistics for each study } & \multicolumn{3}{|c|}{$\mathrm{HR}$ and $95 \% \mathrm{Cl}$} \\
\hline & & HR & Lower limit & Upper limit & & & \\
\hline Liang et al $^{14}$ & Acute myocardial infarction & 1.67 & 1.15 & 2.42 & & | & | \\
\hline Becker et al $^{12}$ & Ischemic stroke & 1.46 & 1.03 & 2.07 & & & \\
\hline \multirow[t]{4}{*}{ Huang et $\mathrm{al}^{13}$} & Ischemic stroke & 2.37 & 1.92 & 2.93 & & & \\
\hline & & 1.84 & 1.34 & 2.54 & & & \\
\hline & & & & 0.01 & 0.1 & 10 & 100 \\
\hline & & & & & Less CVI & More C & \\
\hline
\end{tabular}

Figure 3 Forest plot illustrating the risk of cerebrovascular disease/CVD among PwP from cohort studies. Abbreviations: CVD, cardiovascular disease; PwP, people with Parkinson's disease.

excessive ROS - may attribute to the positive association between PD and CVD: oxidative damage, simultaneously, results in the degeneration of dopaminergic neurons and atherosclerosis, which increases the risk of CVD.

Some molecular targets also contribute to both the diseases. The pathological aggregation of protein $\alpha$-synuclein in PD is also responsible for the poststroke neuronal damage. Animal models with knockdown or knockout of $\alpha$-synuclein demonstrated decreased infarction and better neurological recovery after ischemia. ${ }^{21}$ On the other hand, matrix metalloproteinases (MMPs) are a group of proteins which play an important role in the degradation of extracellular matrix components, remodeling of tissues, shedding of cell surface receptors, and processing of various signaling molecules. CVD may lead to vascular endothelial cell injury, which causes the release of proinflammatory cytokines and free radicals at the neurovascular unit and subsequently activates MMPs. ${ }^{22}$ The activated MMPs not only lead to degradation of extracellular matrix and blood-brain barrier disruption, which is associated with microglial activation and dopaminergic neurodegeneration in PD, but also affect the cleavage of $\alpha$-synuclein, which facilitates the aggregated formation of $\alpha$-synuclein. ${ }^{23}$

The symptoms of PD are not limited to motor dysfunctions but also extend to nonmotor dysfunctions. ${ }^{24}$ Autonomic dysfunction is prevalent among PwP, and orthostatic hypotension $(\mathrm{OH})$ is one of the phenomena of autonomic dysfunction in $\mathrm{PD}$, which presents both before and after the onset of motor symptoms..$^{25,26} \mathrm{OH}$ significantly increases the risk of CVD, which may result from the drop of blood pressure and reduced brain perfusion during postural change. ${ }^{27-29}$ In addition, levodopa is widely prescribed for PwP for the symptomatic relieving, and one of the adverse effects of levodopa is hypotension, which augments $\mathrm{OH} .{ }^{30}$
Some minor factors attribute to the increased risk of CVD for PwP. Due to motor (rigidity and gait disturbance) and nonmotor (apathy, depression, and dementia) symptoms, PwP tend to have a sedentary life, ${ }^{31}$ which is recognized as a risk factor of CVD. ${ }^{32-34}$ In addition, prescription of levodopa increases serum homocysteine level, ${ }^{35}$ which induces atherosclerosis. ${ }^{36}$ Conventional ergot-derived dopamine agonists increase the risk of cardiac valvulopathy, ${ }^{37}$ which may induce the generation of thrombus and embolic stroke. Among PwP with dementia, atypical antipsychotics may be prescribed to manage the behavioral problems, which are also known to increase cardiovascular mortality. ${ }^{38}$ These drug-related complications can be partly attributed to the higher risk of CVD among PwP in their later life.

The present meta-analysis had certain limitations. The small number of studies included in this study was a weak point. Some articles favoring the negative or neutral association of PD with CVD were not included in the analysis because of either unclear definition of $\mathrm{PD}^{39-41}$ or no inclusion of real control patients. ${ }^{42}$ If those omitted studies were included in the clinical case-control studies group for analysis, there would be no association between PD and CVD (Figure S1). However, it is very clear to identify the trend that the later the studies, the higher the association between the two diseases. The change may have resulted from either the utilization of validated diagnostic criteria of $\mathrm{PD}$, which increases the diagnostic accuracy, or the introduction of new concepts about cerebral multimorbidity in the aging brain. In another study, pathological accumulation of proteins (amyloid- $\beta$, tau, $\alpha$-synuclein, and TDP-43) and vascular pathology were simultaneously detected, which indicated the synergic effect between all of them. ${ }^{43}$ With this knowledge, clinicians will not rule out the PD diagnosis based on 
the presence of nonsignificant vascular insults in the brain. Heterogeneity of the included studies is another issue. In the clinical case-control studies, the definition of CVD varied between clinical and radiological findings, which introduced inevitable heterogeneity into the meta-analysis. All the cohort studies were large-scale and population-based, and they had problems in common, such as lack of information on lifestyle or smoking habits and the possibility of the misclassification of PD. Finally, the association between PD and CVD was not able to translate to the management of both the diseases directly. For instance, even if levodopa results in $\mathrm{OH}$ and elevated homocysteine, which may increase the risk of CVD, it is still the most potent and non-replaceable symptomatic treatment for PD. However, the strength of this study was that the meta-analysis method was employed to provide new evidence about the controversy over whether PD is positively associated with CVD. After pooling the information, this study demonstrated that PwP were more likely to have a CVD history and were at a higher risk of future CVD, which necessitates educating PwP on CVD prevention.

In summary, $\mathrm{PD}$ is associated with CVD, which may stem from shared common pathogeneses, or certain nonmotor symptoms or treatment of PD. Although the PwP have fewer vascular risk factors and a lower smoking rate, CVD risk-modification therapy, such as antiplatelet prescription, lipid-lowering treatment, and blood glucose control, should be more emphasized. Moreover, PD-specific vascular risk factors, such as $\mathrm{OH}$ and drug-related complications, should also be considered by movement specialists.

\section{Disclosure}

The authors report no conflicts of interest in this work.

\section{References}

1. de Lau LM, Breteler MM. Epidemiology of Parkinson's disease. Lancet Neurol. 2006;5(6):525-535.

2. Schapira AH, Jenner P. Etiology and pathogenesis of Parkinson's disease. Mov Disord. 2011;26(6):1049-1055.

3. Hughes AJ, Daniel SE, Kilford L, Lees AJ. Accuracy of clinical diagnosis of idiopathic Parkinson's disease: a clinico-pathological study of 100 cases. J Neurol Neurosurg Psychiatry. 1992;55(3):181-184.

4. Korczyn AD. Vascular parkinsonism - characteristics, pathogenesis and treatment. Nat Rev Neurol. 2015;11(6):319-326.

5. De Michele G, Filla A, Volpe G, et al. Environmental and genetic risk factors in Parkinson's disease: a case-control study in southern Italy. Mov Disord. 1996;11(1):17-23.

6. Liou HH, Tsai MC, Chen CJ, et al. Environmental risk factors and Parkinson's disease: a case-control study in Taiwan. Neurology. 1997;48(6):1583-1588.

7. Scigliano G, Musicco M, Soliveri P, Piccolo I, Ronchetti G, Girotti F. Reduced risk factors for vascular disorders in Parkinson disease patients: a case-control study. Stroke. 2006;37(5):1184-1188.
8. Jellinger KA. Prevalence of cerebrovascular lesions in Parkinson's disease. A postmortem study. Acta Neuropathol. 2003;105(5):415-419.

9. Jellinger KA. Prevalence of vascular lesions in dementia with Lewy bodies. A postmortem study. JNeural Transm (Vienna). 2003;110(7):771-778.

10. Jellinger KA. Prevalence of stroke in Parkinson's disease. Mov Disord. 2003;18(6):723-724.

11. Mastaglia FL, Johnsen RD, Kakulas BA. Prevalence of stroke in Parkinson's disease: a postmortem study. Mov Disord. 2002;17(4):772-774.

12. Becker C, Jick SS, Meier CR. Risk of stroke in patients with idiopathic Parkinson disease. Parkinsonism Relat Disord. 2010;16(1):31-35.

13. Huang YP, Chen LS, Yen MF, et al. Parkinson's disease is related to an increased risk of ischemic stroke - a population-based propensity score-matched follow-up study. PLoS One. 2013;8(9):e68314.

14. Liang HW, Huang YP, Pan SL. Parkinson disease and risk of acute myocardial infarction: a population-based, propensity score-matched, longitudinal follow-up study. Am Heart J. 2015;169(4):508-514.

15. Hernan MA, Logroscino G, Garcia Rodriguez LA. Nonsteroidal antiinflammatory drugs and the incidence of Parkinson disease. Neurology. 2006;66(7):1097-1099.

16. Patel M, Coutinho C, Emsley HC. Prevalence of radiological and clinical cerebrovascular disease in idiopathic Parkinson's disease. Clin Neurol Neurosurg. 2011;113(10):830-834.

17. Skeie GO, Muller B, Haugarvoll K, Larsen JP, Tysnes OB. Parkinson disease: associated disorders in the Norwegian population based incident ParkWest study. Parkinsonism Relat Disord. 2013;19(1):53-55.

18. Song IU, Lee JE, Kwon DY, Park JH, Ma HI. Parkinson's disease might increase the risk of cerebral ischemic lesions. Int J Med Sci. 2017;14(4):319-322.

19. Yu EP, Bennett MR. The role of mitochondrial DNA damage in the development of atherosclerosis. Free Radic Biol Med. 2016;100:223-230.

20. Madamanchi NR, Runge MS. Mitochondrial dysfunction in atherosclerosis. Circ Res. 2007;100(4):460-473.

21. Kim T, Mehta SL, Kaimal B, Lyons K, Dempsey RJ, Vemuganti R. Poststroke induction of alpha-synuclein mediates ischemic brain damage. J Neurosci. 2016;36(26):7055-7065.

22. Lakhan SE, Kirchgessner A, Tepper D, Leonard A. Matrix metalloproteinases and blood-brain barrier disruption in acute ischemic stroke. Front Neurol. 2013;4:32.

23. Brkic M, Balusu S, Libert C, Vandenbroucke RE. Friends or foes: matrix metalloproteinases and their multifaceted roles in neurodegenerative diseases. Mediators Inflamm. 2015;2015:620581.

24. Chaudhuri KR, Healy DG, Schapira AH. Non-motor symptoms of Parkinson's disease: diagnosis and management. Lancet Neurol. 2006;5(3):235-245.

25. Goldstein DS. Orthostatic hypotension as an early finding in Parkinson's disease. Clin Auton Res. 2006;16(1):46-54.

26. Velseboer DC, de Haan RJ, Wieling W, Goldstein DS, de Bie RMA. Prevalence of orthostatic hypotension in Parkinson's disease: a systematic review and meta-analysis. Parkinsonism Relat Disord. 2011;17(10):724-729.

27. Novak V, Novak P, Spies JM, Low PA. Autoregulation of cerebral blood flow in orthostatic hypotension. Stroke. 1998;29(1):104-111.

28. Eigenbrodt ML, Rose KM, Couper DJ, Arnett DK, Smith R, Jones D. Orthostatic hypotension as a risk factor for stroke: the Atherosclerosis Risk in Communities (ARIC) study, 1987-1996. Stroke. 2000;31(10):2307-2313.

29. Rose KM, Tyroler HA, Nardo CJ, et al. Orthostatic hypotension and the incidence of coronary heart disease: the Atherosclerosis Risk in Communities study. Am J Hypertens. 2000;13(6 Pt 1):571-578.

30. Mosnaim AD, Abiola R, Wolf ME, Perlmuter LC. Etiology and risk factors for developing orthostatic hypotension. Am J Ther. 2010;17(1):86-91.

31. van Nimwegen M, Speelman AD, Hofman-van Rossum EJM, et al. Physical inactivity in Parkinson's disease. J Neurol. 2011;258(12):2214-2221.

32. Leon-Latre M, Moreno-Franco B, Andres-Esteban EM, et al. Sedentary lifestyle and its relation to cardiovascular risk factors, insulin resistance and inflammatory profile. Rev Esp Cardiol (EnglEd). 2014;67(6):449-455. 
33. Rodriguez Artalejo F, Guallar-Castillon P, Gutierrez-Fisac JL, Ramon Banegas J, del Rey Calero J. Socioeconomic level, sedentary lifestyle, and wine consumption as possible explanations for geographic distribution of cerebrovascular disease mortality in Spain. Stroke. 1997;28(5):922-928.

34. Warren TY, Barry V, Hooker SP, Sui X, Church TS, Blair SN. Sedentary behaviors increase risk of cardiovascular disease mortality in men. Med Sci Sports Exerc. 2010;42(5):879-885.

35. Rogers JD, Sanchez-Saffon A, Frol AB, Diaz-Arrastia R. Elevated plasma homocysteine levels in patients treated with levodopa: association with vascular disease. Arch Neurol. 2003;60(1):59-64.

36. Temple ME, Luzier AB, Kazierad DJ. Homocysteine as a risk factor for atherosclerosis. Ann Pharmacother. 2000;34(1):57-65.

37. Antonini A, Poewe W. Fibrotic heart-valve reactions to dopamine-agonist treatment in Parkinson's disease. Lancet Neurol. 2007;6(9):826-829.
38. Raedler TJ. Cardiovascular aspects of antipsychotics. Curr Opin Psychiatry. 2010;23(6):574-581.

39. Struck LK, Rodnitzky RL, Dobson JK. Stroke and its modification in Parkinson's disease. Stroke. 1990;21(10):1395-1399.

40. Levine RL, Jones JC, Bee N. Stroke and Parkinson's disease. Stroke. 1992;23(6):839-842.

41. Nataraj A, Rajput AH. Parkinson's disease, stroke, and related epidemiology. Mov Disord. 2005;20(11):1476-1480.

42. Korten A, Lodder J, Vreeling F, Boreas A, van Raak L, Kessels F. Stroke and idiopathic Parkinson's disease: does a shortage of dopamine offer protection against stroke? Mov Disord. 2001;16(1):119-123.

43. Jellinger KA, Attems J. Challenges of multimorbidity of the aging brain: a critical update. J Neural Transm (Vienna). 2015;122(4):505-521. 


\section{Supplementary materials}

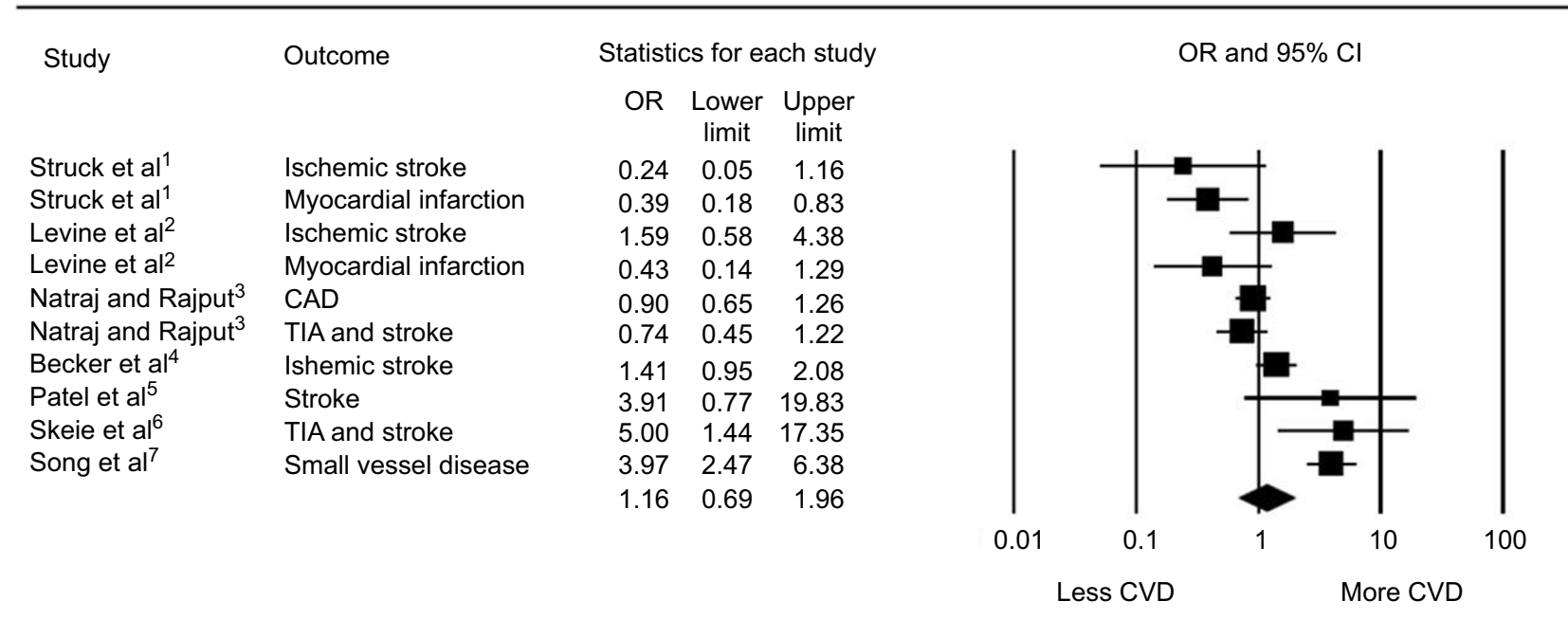

Figure SI Forest plot illustrating the risk of cerebrovascular disease/CVD among PwP from clinical case-control studies, including all the studies with either clear or unclear diagnostic criteria of PD.

Abbreviations: CVD, cardiovascular disease; PwP, people with Parkinson's disease; PD, Parkinson's disease; CAD, coronary artery disease; TIA, transient ischemic attack.

Table SI List of search keywords

\begin{tabular}{ll}
\hline Disease & Keywords \\
\hline PD & Parkinson's Disease[Title/Abstract] OR Parkinson Disease[Title/Abstract] OR Idiopathic Parkinson's Disease[Title/Abstract] OR \\
Idiopathic Parkinson Disease[Title/Abstract] & Cardiovascular disease[Title/Abstract] OR cardiovascular event[Title/Abstract] OR coronary disease[Title/Abstract] OR coronary \\
ischemia[Title/Abstract] OR coronary ischaemia[Title/Abstract] OR coronary artery disease[Title/Abstract] OR coronary heart \\
disease[Title/Abstract] OR myocardial infarction[Title/Abstract] OR heart infarction[Title/Abstract] OR stroke[Title/Abstract] OR \\
cerebrovascular disease[Title/Abstract] OR cerebrovascular event[Title/Abstract] OR cerebrovascular ischemia[Title/Abstract] \\
OR cerebrovascular ischaemia[Title/Abstract] OR cerebrovascular infarction[Title/Abstract] OR brain infarction[Title/Abstract] \\
OR brain ischemia[Title/Abstract] OR brain ischaemia[Title/Abstract] OR transient ischemic attack[Title/Abstract] OR transient \\
ischaemic attack[Title/Abstract] OR peripheral artery disease[Title/Abstract] OR peripheral artery occlusive disease[Title/Abstract]
\end{tabular}

Abbreviations: PD, Parkinson's disease; CVD, cardiovascular disease.

\section{References}

1. Struck LK, Rodnitzky RL, Dobson JK. Stroke and its modification in Parkinson's disease. Stroke. 1990;21(10):1395-1399.

2. Levine RL, Jones JC, Bee N. Stroke and Parkinson's disease. Stroke. 1992;23(6):839-842.

3. Nataraj A, Rajput AH. Parkinson's disease, stroke, and related epidemiology. Mov Disord. 2005;20(11):1476-1480.

4. Becker C, Jick SS, Meier CR. Risk of stroke in patients with idiopathic Parkinson disease. Parkinsonism Relat Disord. 2010;16(1):31-35.
5. Patel M, Coutinho C, Emsley HC. Prevalence of radiological and clinical cerebrovascular disease in idiopathic Parkinson's disease. Clin Neurol Neurosurg. 2011;113(10):830-834.

6. Skeie GO, Muller B, Haugarvoll K, Larsen JP, Tysnes OB. Parkinson disease: associated disorders in the Norwegian population based incident ParkWest study. Parkinsonism Relat Disord. 2013;19(1):53-55.

7. Song IU, Lee JE, Kwon DY, Park JH, Ma HI. Parkinson's disease might increase the risk of cerebral ischemic lesions. Int J Med Sci. 2017;14(4):319-322.
Clinical Epidemiology

\section{Publish your work in this journal}

Clinical Epidemiology is an international, peer-reviewed, open access, online journal focusing on disease and drug epidemiology, identification of risk factors and screening procedures to develop optimal preventative initiatives and programs. Specific topics include: diagnosis, prognosis, treatment, screening, prevention, risk factor modification,

Submit your manuscript here: https://www.dovepress.com/clinical-epidemiology-journal

\section{Dovepress}

systematic reviews, risk and safety of medical interventions, epidemiology and biostatistical methods, and evaluation of guidelines, translational medicine, health policies and economic evaluations. The manuscript management system is completely online and includes a very quick and fair peer-review system, which is all easy to use. 